Pedagogía y Saberes No. 51

Universidad Pedagógica Nacional

Facultad de Educación. 2019. pp. 67-74

\title{
La racionalidad neoliberal y la transformación estructural de la universidad*
}

Artículo de reflexión

Neoliberal Rationality and the Structural Transformation of the University

A racionalidade neoliberal e a transformação estrutural da universidade

Alexandre Alves**

Para citar este artículo:

Alves, A. (2019). La racionalidad neoliberal y la transformación estructural de la universidad. Pedagogía y Saberes, 51, 67-74.

* Este artículo se deriva de una investigación en curso financiada por cAPEs (Coordenação de Aperfeiçoamento de Pessoal de Nível Superior)

** Profesor de la Universidade do Vale do Rio dos Sinos (Unisinos), Doctor en Historia de la Universidade de São Paulo (USP) e investigador del Grupo de Estudos e Pesquisa em Inclusão (GEPI).

Correo electrónico: sandoralves@gmail.com

Código ORCID: orcid.org/0000-0002-0810-650X 


\title{
Resumen
}

El objetivo de este texto derivado de una investigación en curso es proponer una reflexión sobre el futuro de la universidad, a partir de la problematización del impacto de la racionalidad neoliberal en las formas de producción del conocimiento y de organización de la vida académica. Para desarrollar este análisis, se reconstruyen brevemente las principales transformaciones de la universidad moderna desde su advenimiento con la fundación de la Universidad de Berlín por Wilhelm von Humboldt, en 1810, quien creó el modelo de la universidad de investigación, hasta la emergencia del modelo de la universidad neoliberal. El propósito del artículo es destacar las racionalidades que orientan la transformación de la universidad a lo largo del tiempo. Con ese análisis histórico se intenta demostrar que ningún modelo puede ser considerado natural, absoluto, inevitable, innegociable y que la universidad como institución está habitada por una mezcla de racionalidades contradictorias. Para concluir, se usa un ensayo de Derrida (2001) para mostrar que la adopción no contestada del modelo neoliberal puede destruir la autonomía en la investigación y enseñanza que hasta hoy caracterizó a la universidad moderna, y compromete su misión en el futuro.

\section{Palabras clave}

neoliberalismo; Bildung; capitalismo académico; Humanidades

\begin{abstract}
The aim of this text, derived from an investigation, is to propose a reflection on the future of the university, from the problematization of the neoliberal rationality impact on the forms of knowledge production and organization of academic life. To develop this analysis, the main transformations of the modern university since its advent with the founding of the Berlin University by Wilhelm von Humboldt, in 1810, who created the model of the research university, until the emergence of the neoliberal university model. The purpose of the article is to highlight the rationalities that govern the transformation of the university over time. This historical analysis attempts to demonstrate that no model can be considered natural, absolute, inevitable, non-negotiable, and that and that the university as an institution is inhabited by a mixture of contradictory rationalities. To conclude, the Derrida's essay (2001), is used to show that the uncontested adoption of the neoliberal model can destroy the autonomy of research and teaching that until today characterized the modern university, and compromise its mission in the future.
\end{abstract}

\section{Keywords}

neoliberalism; Bildung; academic capitalism; Humanities

\section{Resumo}

O objetivo deste texto derivado de uma pesquisa em andamento é propor uma reflexão sobre o futuro da universidade, a partir da problematização do impacto da racionalidade neoliberal nas formas de produção do conhecimento e de organização da vida acadêmica. Para desenvolver esta análise, reconstroe-se brevemente as principais transformações da universidade moderna desde seu advento com a fundação da Universidade de Berlim por Wilhelm von Humboldt, em 1810, que criou o modelo da universidade de pesquisa, até a emergência do modelo da universidade neoliberal. 0 propósito do artigo é destacar as racionalidades que presidem a transformação da universidade ao longo do tempo. Com essa análise histórica intenta-se demonstrar que nenhum modelo pode ser considerado natural, absoluto, inevitável, inegociável e que a universidade, como instituição é habitada por uma mistura de racionalidades contraditórias. Para concluir, usa-se um ensaio de Derrida (2001) para mostrar que a adoção não contestada do modelo neoliberal pode destruir a autonomia de pesquisa e ensino, que até hoje caracterizou a universidade moderna, e compromete sua missão no futuro.

\section{Palavras-chave}

neoliberalismo; Bildung; capitalismo acadêmico; Humanidades 


\section{Introducción}

La universidad moderna es una institución en crisis y su papel en el mundo actual ha sido puesto en cuestión de forma constante (Thayer, 2002). Algunos se inclinan a atribuir esta crisis a los cambios tecnológicos por los que pasamos en las últimas décadas. Sin embargo, las consecuencias disruptivas provocadas por la revolución digital y por el advenimiento de la llamada Cuarta Revolución Industrial apenas aceleran transformaciones económicas y sociales resultantes de la generalización de modos neoliberales de gobierno de la sociedad. Según Klaus Schwab (2016), la emergencia del nuevo paradigma tecnocientífico e industrial, caracterizado por la automatización creciente, la interconexión generalizada y la fusión de diferentes tecnologías, creó un escenario en el que los puestos de trabajo y sectores enteros de actividad humana corren el riesgo de desaparecer en un futuro próximo. Los gestores universitarios y docentes ya observan con inquietud la producción de un enorme contingente de portadores de títulos y diplomas universitarios que no consigue emplearse en las áreas y especialidades para los cuales se formaron y acaban constituyendo parte del creciente número de trabajadores en ocupaciones precarias, el llamado precariato. Sin embargo, problemas sociales como precarización y desempleo no pueden ser atribuidos solo a los cambios tecnológicos, como si estos implicaran un destino ineludible, pero sobre todo a formas neoliberales de organización de la sociedad y de gestión del trabajo. ${ }^{1}$

Movidos por las presiones del mercado globalizado, muchos defienden como solución para la crisis la flexibilización de la estructura de la universidad, con base en principios adoptados por las empresas privadas como efectividad, performatividad y competencia. Para alcanzar "calidad" y "excelencia" - palabras clave de ese discurso-, a partir de los años 1980, agencias internacionales como la oCDE y el Banco Mundial pasaron a defender la adopción del New Public Management (NPM) en la enseñanza superior (Giroux, 2014). El NPM se basa en las teorías del capital humano y en los sistemas estandarizados de evaluación en masa para crear un "mercado académico" e introducir en la universidad criterios empresariales de gestión y organización. Este cambio dio origen a prácticas como los rankings universitarios, el productivismo académico, los índices de impacto,

1 En su libro L'école n'est pas une entreprise, Christian Laval (2004) ya constataba la degradación del vínculo entre diploma y empleo como consecuencia del capitalismo neoliberal, que valora las competencias más cercanas a las situaciones profesionales y desvaloriza en la misma medida la fuerza simbólica del título académico. la competición por fondos entre institutos y departamentos, así como la reorientación de la investigación para fines inmediatos y productos útiles.

El objetivo de este texto es proponer una reflexión sobre el futuro de la universidad a partir de la problematización del impacto de la racionalidad neoliberal en las formas de producción del conocimiento y de organización de la vida académica. Mi propósito no es investigar sistemas de enseñanza o instituciones determinados, sino destacar las racionalidades que presiden la transformación de la universidad como institución a lo largo del tiempo. Pretendo demostrar, con ese análisis histórico, que ningún modelo puede ser considerado natural, absoluto, inevitable o innegociable y que hay que rediscutir urgentemente la misión de la universidad en los días actuales.

\section{Ascensión y caída del modelo humboldtiano de universidad}

Para desarrollar este análisis, comienzo por reconstruir con brevedad las principales transformaciones de la universidad moderna desde su advenimiento con la fundación de la Universidad de Berlín por Wilhelm von Humboldt, en 1810, que creó el modelo de la universidad de investigación. La idea central que llevó a la fundación de la universidad de Berlín es la de Bildung como formación integral del ser humano (Alves, 2019; Assmann, 1994; Bollenbeck, 2012; Vierhaus, 2004). Bildung es la formación de la totalidad de la persona humana como fin en sí mismo. Como dice Reinhart Koselleck en su análisis del tema, "toda Bildung es Selbstbildung [autoformación] y, así, señala una tarea que corresponde única y exclusivamente al individuo" (Koselleck, 1990, p. 20). Esta tarea no depende de nada exterior, sin embargo, exige situaciones y circunstancias que deben ocurrir las cuales, en la visión de Humboldt, serían proporcionadas por una institución vista como comunidad de investigadores y estudiantes con plena autonomía intelectual y administrativa: la universidad.

Según Koselleck, las interpretaciones que asocian a Bildung con trazos como introversión, pasividad y apolitismo solo caricaturizan un concepto bastante complejo. Como un desarrollo pleno de todos los lados de la persona humana, Bildung no lleva a la contemplación pasiva de la alta cultura, sino que obliga al individuo a actuar en el mundo, a comunicarse y comprometer toda su energía por el bien de la sociedad. Estímulo a la vita activa y no al cultivo egoísta de la interioridad. Por eso, la sociabilidad es uno de sus elementos constitutivos (Sorkin, 1983). 
Es en la universidad, tal como ha sido concebida por Humboldt, que esa sociabilidad podría desarrollarse con libertad.

Entre el último cuarto del siglo XVIII y el primero del siglo xIX, ese ideal educativo tuvo una función emancipadora y estaba fuertemente asociado a ideas como la independencia de toda autoridad externa (iglesias, Estado, partidos, etc.), la liberación de las jerarquías estamentales, que en esa época aún regulaban las relaciones sociales en Alemania, y el rechazo de los preceptos y dogmas teológicos, tanto protestantes como católicos. Así, el proyecto de la universidad moderna, que a lo largo del siglo XIX se expandiría hacia diversos países en todo el mundo, tenía en su centro las ideas de totalidad, formación y autonomía (Rüegg, 2004). La universidad humboldtiana fue, en efecto, la institucionalización del ideal de Bildung, en la forma de una institución responsable del fomento y la producción de una cultura y de un conocimiento libre de presiones externas, que en la época venían ante todo del Estado y de la religión. Según Humboldt (1997):

Con relación a la idea general de la universidad [...] el Estado no puede exigir de las instituciones científicas superiores nada que se relacione inmediata o directamente a sí mismo. Por el contrario, debe comprender que, al alcanzar su auténtica finalidad, éstas también cumplen las finalidades del propio Estado. Las finalidades del Estado, así, alcanzan un nivel superior de realización. Y un nivel que, por sí solo, el Estado no sería capaz de alcanzar. (pp. 88-89).

En el modelo concebido por Humboldt, la autonomía administrativa e intelectual del cuerpo universitario frente a los poderes externos es un elemento central, sin el cual no se puede hablar de una "institución científica superior". Mientras las escuelas solo transmiten el saber validado y legitimado por la tradición, las universidades tienen la obligación de crear y producir conocimiento nuevo. La investigación es necesaria porque la ciencia es una búsqueda infinita y solo la plena libertad intelectual asegura el avance continuo del conocimiento. En el modelo humboldtiano, la universidad debería no solo garantizar la formación integral de todos los aspectos de la persona humana, sino también promover la totalización y la síntesis de los nuevos conocimientos. Esta síntesis debería ser producida por la unidad orgánica entre enseñanza e investigación y por el papel central desempeñado por la facultad de filosofía, que debería ser el foco de difusión de las luces y del conocimiento racional. Según Kant:
Importa absolutamente que en la universidad se dé a la comunidad erudita una Facultad que, independientemente de las órdenes del gobierno en cuanto a sus doctrinas, tenga la libertad, no de proferir órdenes, pero al menos de juzgar todas las que tienen que ver con el interés de la ciencia, es decir, con el de la verdad, en que la razón debe estar autorizada a hablar públicamente; porque, sin semejante libertad, la verdad no vendría a la luz (para daño del propio gobierno), pero la razón es libre por su naturaleza y no acoge ninguna orden para aceptar algo como verdadero. (Kant, 1798, citado en Brandt, 2017, p. 46).

Sin embargo, en la medida en que las ciencias se dividieron en una infinidad de disciplinas y subdisciplinas y la sociedad se ha vuelto más compleja y diferenciada, esa síntesis se volvió problemática. El modelo de la universidad moderna humboldtiana fue pensado desde una perspectiva filosófica idealista que simplifica demasiado las complejas conexiones entre ciencia y sociedad. En las palabras de Habermas (2003):

En el momento de nacimiento de la universidad clá-
sica alemana, los reformadores prusianos dibujaron
una imagen que sugería un nexo muy simplificado
entre los procesos de aprendizaje científico y las
formas de vida de las sociedades modernas. Desde
el punto de vista de una filosofía idealista de la
reconciliación, ellos han exigido de la universidad
una fuerza de totalización que, desde el principio,
sería necesariamente una sobrecarga para esa
institución. (p. 86).

Aunque el modelo humboldtiano persiste, a principios del siglo $\mathrm{xx}$ hay una reconfiguración global de la universidad con la emergencia de nuevos modelos. Max Weber vio muy bien la reestructuración de la universidad en curso de acuerdo con el modelo norteamericano de la universidad de investigación. En su conferencia "Ciencia como vocación", Weber constataba la imposibilidad del modelo humboldtiano en una sociedad diferenciada en términos funcionales y ya veía la nueva misión de la universidad: la formación del "especialista" (Waizbort, 2015). El Estado y la economía moderna necesitaban una serie de profesionales altamente cualificados. Así, el modelo de la formación daba lugar al de la profesionalización. La consecuencia práctica de ello, en el plano organizacional, fue la división de la universidad en una serie de institutos descentralizados, cada uno concebido para promover una especialidad a través de la enseñanza y la investigación. La vida universitaria se burocratiza y ninguna "facultad" puede clamar más el papel de totalidad o síntesis. 
La crisis del modelo humboldtiano da lugar a una reorganización de la universidad como una gran empresa controlada por la burocracia estatal (Ringer, 2000). Son las nuevas necesidades del capitalismo de Estado las que impulsan esos cambios, como observó Weber (2013) en su famosa conferencia, ya mencionada, "Ciencia como vocación":

Los grandes institutos de medicina o de ciencias naturales son empresas del 'capitalismo estatal'. No pueden ser administrados sin medios de producción en enorme escala. Y entonces se manifiesta la misma situación que uno observa en toda parte donde la actividad capitalista se ha introducido: la 'separación del trabajador en relación a los medios de producción'. El trabajador, el Assistent, por lo tanto, depende de los materiales puestos a disposición por el Estado; en consecuencia, se queda tan dependiente del director del instituto cuanto un empleado en un taller, pues el director del instituto se cree, con absoluta buena fe, que aquello es 'su' instituto, y así lo comanda. Y muchas veces se encuentra en una situación de precariedad análoga a la de cualquier sujeto 'proletarizado' y a la del assistant de la universidad americana. (p. 395).

Con el proceso de burocratización y funcionalización de la universidad, se pierden dos características centrales del modelo humboldtiano: la unidad orgánica entre enseñanza e investigación y la autonomía intelectual y administrativa. No será más misión de la universidad formar un individuo total y promover la síntesis de los conocimientos, sino atender las demandas prácticas de las sociedades modernas por medio de la investigación científica y de la formación de profesionales calificados en las más diversas áreas. Es esa misma exigencia de profundización de la función social de la universidad la que lleva a la expansión creciente del acceso a la universidad y, también, a la diferenciación de los sistemas de enseñanza superior en los años de la posguerra, más aceleradamente desde los años 1960.

\section{"Capitalismo académico" y racionalidad neoliberal}

La constante expansión de la enseñanza superior en los años de la posguerra, junto con el advenimiento de las teorías del capital humano, llevaron a cambios estructurales en las universidades. En línea con estas teorías, la investigación y la formación proporcionadas por las universidades pasan a ser vistas como factores de producción esenciales para el funcionamiento de las economías de mercado. Como ya anticipó Weber, las universidades se asemejan cada vez más a las empresas y se someten a una división de funciones y a patrones de productividad y eficiencia provenientes de la empresa capitalista.

El énfasis se desplaza de modo paulatino de la formación del especialista hacia la masificación de títulos. Formar un especialista es un proceso caro y lento. Así, a partir de la década de 1980, con la internacionalización de la economía y la adopción del discurso neoliberal de la gestión por gobiernos e instituciones como el Banco Mundial y el FMI, se produce una reestructuración de las universidades en las que la investigación y la enseñanza se separan. Una parte significativa de la investigación "aplicada" pasa a ser hecha fuera de las universidades. Dentro de ellas, se forman centros de "excelencia", íntimamente vinculados al mercado, que abdican de la tarea de formación. Las facultades, a su vez, titulan en masa trabajadores flexibles para un mercado de trabajo inestable. La especialización pasa a concentrarse en los programas de posgrado, los cuales son sometidos cada vez más a choques de gestión con la imposición de criterios para garantizar "competitividad", "calidad", "eficiencia" y "excelencia". El problema es que las nuevas técnicas de gestión del "capitalismo académico" limitan la autonomía intelectual y organizacional de la universidad de forma radical y hacen que las universidades se asemejen cada vez más a las empresas privadas a la vez que impulsan la simbiosis con el mercado.

En el libro La universidad en ruinas, Bill Readings (1996) atacó lo que llamó "universidad corporativa" (enterpreneurial university): la universidad redefinida como un emprendimiento en el que lo que importa sobre todo es la inversión de capital y el retorno financiero. A partir de los años 1980, las universidades norteamericanas y europeas son reestructuradas y pasan a ser modeladas por la lógica y la dinámica del mercado. Todos los elementos del currículo se transforman en mercancías sujetas a la fluctuación de la oferta y la demanda, lo que conduce a la devaluación creciente de la investigación básica y de las humanidades. La universidad se reorienta hacia la formación de especialistas para actividades orientadas al lucro a corto plazo: obtención de patentes y derechos de autor (copyrights), gestación de empresas emergentes (startups) y todo tipo de productos susceptibles de generar retorno comercial, como los cursos en línea. Las reformas universitarias son impulsadas por el aumento de la dependencia de la financiación privada, con la reducción de recursos estatales, y por la creciente interacción entre universidad e industria. Como consecuencia, se forma una verdadera simbiosis entre universidad y mercado, hasta el punto de que se vuelve difícil distinguir sus fronteras. El modelo neoliberal subordina íntegramente 
la universidad a las demandas del mercado, y la transforma en una incubadora de empresas y a ella misma en un tipo de empresa (Ward, 2012).

El problema es que el modelo de la universidad corporativa o neoliberal, con su simbiosis creciente entre educación y mercado, reduce la autonomía intelectual y administrativa de las universidades y de su cuerpo docente, exponiéndolas cada vez más a las presiones económicas y a las fluctuaciones de los mercados. Así, la preocupación por la formación de la persona humana y el debate crítico pasan a un segundo plano. No sorprende que se haya hablado de esa reestructuración como "ruina" o "destrucción" de la universidad.

De acuerdo con el nuevo discurso, la universidad se ve cada vez más como una fuerza productiva y las técnicas de gestión apuntan a garantizar la mayor eficiencia en la reproducción de capital humano con los menores costos posibles. En el escenario de la llamada "nueva economía", centrada en las nuevas tecnologías de información y comunicación, se impone lo que fue llamado "capitalismo académico" (Slaughter y Rhoades, 2004). Según el modelo del "capitalismo académico", la expansión de la capacidad gerencial es necesaria para supervisar los flujos de recursos y su asignación para inversiones en investigación, personal e infraestructura a fin de generar productos y servicios para el mercado. Por tanto, la propia institución de enseñanza superior, en ese modelo, se presenta como negociante en el mercado, haciendo la intermediación entre la investigación y el sector privado.

Para comprender mejor esta transformación estructural de la universidad y la racionalidad que la preside, tenemos que recurrir a los análisis de Michel Foucault en el curso Nacimiento de la Biopolítica (Foucault, 2004). En ese curso, Foucault analizó la racionalidad neoliberal como generalización de la forma empresa para todo el campo social, y la adopción de la forma mercado como principio de inteligibilidad de los comportamientos individuales y de la acción gubernamental. En este modelo, el trabajador es visto como emprendedor de sí mismo, responsable de la formación, gestión y desarrollo de su propio capital, o sea, de las competencias y habilidades adquiridas en el sistema de enseñanza formal que lo hacen útil y empleable por el mercado. La inversión en capital humano pasa a ser vista como principal fuente de innovación y desarrollo económico. La adopción de nuevas técnicas de gestión en la universidad intentaría, justamente, garantizar la mayor eficiencia con el menor costo posible en la producción y reproducción del capital humano que el mercado y el Estado necesitan para impulsar el desarrollo económico.
En el nuevo modelo de universidad, el estudiante es concebido como un empresario de sí mismo que encara su formación como una inversión pre-profesional. La función de la universidad deja de ser la formación de la personalidad o del especialista y pasa a ser la de fabricar el empresario de sí quien se ha convertido en un modelo de subjetividad y un imperativo existencial omnipresente en las sociedades contemporáneas. El individuo es llamado a actuar sobre sí mismo para perfeccionarse, adaptarse y transformarse de modo continuo, para adquirir las competencias y capacidades que le permitirán valorarse como capital humano y realizar su proyecto de vida. El individuo emprendedor es visto, al mismo tiempo, como proveedor y administrador de ese capital, que debe asumir los riesgos inherentes a su gestión. Así, él debe desarrollar y demostrar en su cotidianidad cualidades como autonomía, flexibilidad, responsabilidad, creatividad, innovación y autoeficacia. En la nueva configuración de la subjetividad, como muestra Bröckling (2016), el imperativo del emprendimiento se convierte en un verdadero campo de fuerza que gobierna la vida, informa el pensamiento y orienta los comportamientos (Bröckling, 2016):

El llamado para actuar como un emprendedor de la
propia vida produce un modelo para que la gente
entienda lo que son y lo que deben ser y les dice
cómo trabajar sobre sí mismas para convertirse en
lo que deben ser. En otras palabras, el self empre-
sarial es una forma de subjetivación. Como tal, la
actividad emprendedora es menos un hecho que
un campo de fuerza. Ella es una meta por la cual los
individuos luchan, una medida según la cual ellos
juzgan su conducta, un ejercicio diario para trabajar
sobre el selfy, por fin, un generador de verdad por
el cual ellos llegan a conocerse a sí mismos. (p. viii).

Al mercado de trabajo de la nueva economía no le interesa más el profesional o especialista con amplia calificación en su área, sino un trabajador dotado de competencias básicas y flexible, lo suficiente para adaptarse a las fluctuaciones de una economía dinámica. La adaptabilidad, flexibilidad, resiliencia, autorregulación, emprendimiento pasan a ser palabras clave del discurso de la nueva gestión universitaria. La universidad, en consecuencia, pasa a ser una fábrica de subjetividades en línea con el nuevo capitalismo. De acuerdo con ese modelo, cada uno debe estar constantemente dispuesto a empezar de nuevo, a recalificarse, pasar por un "reciclaje" profesional y continuar aprendiendo por el resto de la vida. El sujeto emprendedor es aquel que perdió el ethos de su profesión y se presenta como mano de obra disponible para todo tipo de emprendimiento y, al mismo tiempo, se presenta como consumidor de una serie de productos y servicios, en primer lugar, 
los propios servicios educativos (sean cursos universitarios, especializaciones, MBA, cursos a distancia o cursos preparatorios) que prometen recalificarlo para permanecer en el mercado de trabajo, o sea, para mantener el valor de su capital humano.

La imposición de la lógica del mercado a las instituciones de enseñanza superior conduce a los males del productivismo y del inmediatismo (Cowen, 2013). Según el modelo gerencial de la universidad corporativa, la tarea principal de la universidad es prestar servicios a la sociedad (entiéndase: suministrar productos útiles al mercado). La sociedad paga por la enseñanza e investigación realizadas en las universidades y debe recibir, a cambio de esa inversión, un beneficio palpable e inmediato por el menor costo posible. Tal beneficio se traduce en la producción de bienes e innovaciones susceptibles de retorno comercial (provenientes de la investigación) y titulación masiva de estudiantes (provenientes de la enseñanza). Este discurso neoliberal de la accountability, sin embargo, alimenta una falsa idea de bien público y de inclusión. El estudiante es incluido solo como ciudadano-consumidor y recibe de la institución de enseñanza superior no la formación para convertirse en un profesional o especialista, sino para ser un trabajador flexible y un consumidor "informado" (Brown, 2015).

\section{La universidad como esfera pública y lugar de la diferencia}

Los distintos momentos del proceso de modernización han impuesto a la universidad como institución configuraciones específicas de acuerdo con desarrollos históricos, sociales y económicos más amplios. Por eso, ningún modelo universitario puede ser elegido norma supra-histórica de lo que debería ser la universidad. Lo que llamamos universidad corresponde a una realidad siempre cambiante, que se transforma de modo continuo, se estructura y se restructura permanentemente. A pesar de eso, el modelo humboldtiano de la universidad moderna preserva todavía su atracción para intelectuales y estudiantes que se baten en la defensa del espacio de las humanidades y contra la hegemonía global de la universidad neoliberal (Hohendahl, 2011). Sin embargo, el modelo idealista humboldtiano ya no puede desempeñar el papel crítico que tuvo en el pasado, cuando sus ideales de personalidad, autonomía y saber desinteresado ayudaron a sostener, en el plano epistemológico, la lucha de la burguesía por emancipación. Como demostró Jean-François Lyotard (2009), el ideal ilustrado de progreso del conocimiento forma parte de un metarrelato que presupone una filosofía evolucionista de la historia y una concepción universalista del sujeto, que se vuelven problemáticos en tiempos posmodernos. El modelo humboldtiano no corresponde más a las transformaciones en la cultura, en la sociedad y en los modos de producción y legitimación del conocimiento en el capitalismo contemporáneo. Por lo tanto, contraponer el modelo humboltdiano a la universidad neoliberal sería solo un llamamiento nostálgico a un pasado idealizado sin real potencial crítico y cuestionador.

En el ensayo La universidad sin condición, Jacques Derrida (2003) intenta mostrar que la adopción no contestada del modelo neoliberal puede destruir el papel de la universidad como esfera crítica de debate y disensión y comprometer su misión en el mundo actual. Como demuestra Derrida, el papel de la universidad va más allá de los fines inmediatos de acelerar la reproducción del capital e impulsar el metabolismo económico. La universidad es el lugar donde es posible pensar de otros modos, desafiar las interpretaciones dominantes, problematizar las prácticas enraizadas, sacudir aquello que se tiene por evidente y fuera de duda. La universidad, y dentro de ella las humanidades, tiene como tarea esencial poner en cuestión aquello que no se suele cuestionar.

En ese sentido, a diferencia de otras áreas, las humanidades no existen para ofrecer "soluciones" a problemas sociales o económicos, ni para ofrecer una dirección a una sociedad en crisis de valores. Las humanidades problematizan lo que no se veía como problemático, cuestionan lo que se tenía por incuestionable, desorientan más que orientan, y aumentan la incertidumbre en lugar de reducirla. A pesar del cobro creciente por utilidad social, las ciencias humanas no sirven para reducir la inmensa complejidad de la realidad a proposiciones sencillas y fórmulas técnicas. Es solo en la medida en que tienen autonomía para pensar y divergir de los saberes hegemónicos que las humanidades pueden realizar su función crítica. Por eso ya Kant, a finales del siglo xviII, colocaba la "facultad" de filosofía en el centro de la universidad. Las humanidades tenían entonces la función de garantizar la independencia de la universidad de los poderes externos -en la época de Kant, la política y la religión-y la autonomía crítica del pensamiento. Hoy, para mantener su papel crítico en una sociedad marcada por la expansión de la tecnociencia y por el capitalismo financiero, el propio proyecto de autonomía de la universidad tiene que ser reinventado. La transformación de la universidad en fábrica de empresas y reproductor de capital humano, sin embargo, impide esa tarea.

En conclusión, la universidad, según Derrida, debe ser una esfera pública, un espacio crítico de debate, de disensión y de resistencia, donde es posible pensar 
diferente, pensar de otro modo lo que se suele pensar del mismo modo. La simbiosis entre universidad y mercado, sin embargo, al acelerar la producción académica y canalizarla hacia los sectores "aplicados", destruye las propias condiciones para la producción de conocimiento crítico y de calidad. Este tipo de conocimiento requiere tiempo para reflexión, discusión y maduración y no se cuantifica fácilmente, así como su beneficio para la sociedad es a largo plazo y no se mide con facilidad. Así, productivismo, aceleración e inmediatismo comprometen el papel eminentemente social y crítico de la universidad. Por lo tanto, ante las transformaciones estructurales por las que pasa la universidad en el mundo contemporáneo, la tarea que nos cabe es pensarla de otro modo y cuestionar la aparente evidencia con que se toma el advenimiento de la universidad corporativa.

\section{Referencias}

Alves, A. (2019). The german tradition of self-cultivation (Bildung) and its historical meaning. Educação \& Realidade, 44 (2) [en línea]. https://dx.doi. org/10.1590/2175-623683003

Assmann, A. (1994). Construction de la mémoire nationale. Une brève histoire de l'idée allemande de Bildung (Trad. Françoise Laroche). Paris: Maison des sciences de l'homme.

Bollenbeck, G. (2012). Glanz und Elend eines deutschen Deutungsmusters. En H. Hastedt (Ed.), Was ist Bildung? Eine Textanthologie (pp. 155-162). Berlin: Reclam.

Brandt, R. (2017). Die Idee der Universität und „Der Streit der Fakultäten“. En Ludger Honnefelder (Ed.), Kants »Streit der Fakultäten " oder der Ort der Bildung zwischen Lebenswelt und Wissenschaften (pp. 43-65). Weilerswist: Velbrück Wissenschaft.

Bröckling, U. (2016). The entrepreneurial self: fabricating a new type of subject. London: Sage.

Brown, W. (2015). Undoing the demos: Neoliberalism's stealth revolution. Massachusetts: MIT Press.

Cowen, R. (2013). A universidade e atuais desafios. Mercado, mobilidade e performatividade (Trad. Ricardo Maurício Soares Baptista). Curitiba: CRV.

Derrida, J. (2003). A universidade sem condição (Trad. Evando Nascimento). São Paulo: Estação Liberdade.

Foucault, M. (2004). Naissance de la biopolitique. Cours au Collège de France (1978-1979). Paris: Gallimard/Seuil.

Giroux, H. A. (2014). Neoliberalism's war on higher education. Chicago: Heymarket Books.
Habermas, J. (2003). Zeitdiagnosen. Frankfurt: Suhrkamp.

Hohendahl, P. U. (2011). Humboldt revisited: liberal education, university reform, and the opposition to the neoliberal university. New German Critique, 113, 159-196.

Humboldt, W. von. (1997). Sobre a organização interna e externa das instituições científicas superiores em Berlim. En J. Kretschmer y J. C. de C. Rocha (Org.), Um mundo sem universidades? (pp. 79-100). Rio de Janeiro: UERJ.

Koselleck, R. (1990). Einleitung zur anthropologischen und semantischen Struktur der Bildung, En R. Koselleck (Ed.), Bildungsbürgertum im 19. Jahrunderts. Teil II. Bildungsgüter und Bildungswissen (pp. 11-46). Stuttgart: Klet-Cotta.

Laval, C. (2004). L'école n'est pas une entreprise: Le néoliberalisme à l'assaut de l'enseignement public. Paris: La Découverte.

Lyotard, J. (2009). A condição pós-moderna (Trad. Ricardo Corrêa Barbosa). Rio de Janeiro: José Olympio.

Readings, B. (1996). The university in ruins. Cambridge: Harvard University Press.

Ringer, F. (2000). O declínio dos mandarins alemães (Trad. Dinah de Abreu Azevedo). São Paulo: Edusp.

Rüegg, W. (Ed.). (2004). A history of university in Europe [vol. 3]. Cambridge: Cambridge University Press.

Schwab, K. (2016). A quarta revolução industrial. São Paulo: Edipro.

Slaughter, S. y Rhoades, G. (2004). Academic capitalism: politics, policies, and the entrepreneurial university. Baltimore, MD: Johns Hopkins University Press.

Sorkin, D. (1983). Wilhelm von Humboldt: The theory and practice of self-formation (Bildung), 1791-1810. Journal of the History of Ideas, 44 (1), 55-73.

Thayer, W. (2002). A crise não moderna da universidade moderna (Trad. Romulo Monte Alto). Belo Horizonte: UFMG.

Vierhaus, R. (2004). Bildung. En O. Brunner, W. Conze, R. Koselleck (Eds.), Geschichtliche Grundbegriffe. Historisches Lexikon zur politisch-sozialen Sprache in Deutschland, Band 4 (pp. 508-551). Stuttgart: Klett-Cotta.

Waizbort, L. (2015). Formação, especialização, diplomação. Da universidade à instituição de ensino superior, Tempo Social, 27 (2), 45-74.

Ward, S. C. (2012). Neoliberalism and the global restructuring of knowledge and education. Nueva York: Routledge.

Weber, M. (2013). Ciência como vocação. En A. Botelho (Org.), Sociologia Essencial (pp. 392-431). São Paulo: Penguin Classics/Companhia das Letras. 